\title{
Miradas de la crisis contemporánea: la Gran Recesión 2008-2009 **
}

\author{
Views of the contemporary crisis: Great Recession 2008-2009
}

\author{
Jesús Lechuga Montenegro ${ }^{1}$ \\ Faustino Vega Miranda ${ }^{2}$
}

\section{RESUMEN}

En este trabajo se estudian las causas del origen de la crisis 2008-2009, desde tres posiciones teóricas. Primero, la historia económica, cuyo enfoque ayuda a contextualizar el cambio de modelo de un Estado Benefactor a un Estado Neoliberal. Los historiadores consideran que la dinámica del periodo neoliberal se refiere a un evento de largo horizonte de acuerdo a las acciones post crisis y porque no existen caminos alternativos viables contra la teoría que pugna la libertad a ultranza. Segundo, desde la teoría económica convencional la principal causa de la crisis fue la falta de mecanismos de mercado para regular la actividad económica; posición que se contrapone con un enfoque heterodoxo: el Estado debe intervenir en los procesos económicos. Finalmente, la Economía Política Marxista estudia los hechos desde una categoría relativamente reciente, la financiarización, donde el modelo de acumulación se basa en las finanzas. El sector financiero se extiende a casi todos los

\footnotetext{
* Artículo científico cuyo origen surge de la reflexión de las causas y consecuencias de la crisis 2008-2009 a diez años de su aparición.

1 Economista, Universidad Nacional Autónoma de México, México. Maestro en Economía, Escuela Central de Planificación y Estadística, Polonia. Maestro en Economía, Universidad de París X-Nanterre, Francia. Doctor en Ciencias Económicas, Universidad Autónoma Metropolitana, México. Profesor-Investigador del Departamento de Economía, Universidad Autónoma Metropolitana Unidad Azcapotzalco. Correo-e: montenegro@azc.uam.mx

2 Economista, Universidad Nacional Autónoma de México, México. Maestro en Economía, Universidad Nacional Autónoma de México, México. Alumno del doctorado en Pedagogía, Universidad Nacional Autónoma de México, México. Profesor adscrito al Programa de Economía en la Facultad de Estudios Superiores Acatlán, UNAM. Correo-e: kopola9@yahoo.com.mx
}

(C) Los autores. Este artículo es publicado por Pensamiento Crítico de la Facultad de Ciencias Económicas, Universidad Nacional Mayor de San Marcos. Este es un artículo de acceso abierto, distribuido bajo los términos de la licencia Creative Commons Atribucion - No Comercia_Compartir Igual 4.0 Internacional. (http://creativecommons.org/licenses/by-nc-sa/4.0/) que permite el uso no comercial, distribución y reproducción en cualquier medio, siempre que la obra original sea debidamente citada. 
sectores de la vida económica y esa penetración tan abrumadora ha contribuido a la fragilidad del sistema. El objetivo es hacer una lectura de los distintos enfoques, en conjunto, para comprender de manera más adecuada el fenómeno en estudio.

Palabras clave: Gran Recesión; sistema financiero; crisis financiera; regulación; financiarización.

Clasificación JEL: E24, E44, F34, F36, B50.

\section{ABSTRACT}

In this paper we study the causes of the origin of the crisis 2008-2009, from three theoretical positions. First, economic history, whose approach helps to contextualize the change of model from a Benefactor State to a Neoliberal State. Historians consider that the dynamics of the neoliberal period refers to a long-horizon event according to post-crisis actions and because there are no viable alternative paths against the theory that strives for freedom at all costs.Second, from conventional economic theory the main cause of the crisis was the lack of market mechanisms to regulate economic activity; position that is opposed to a heterodox approach: State must intervene in economic processes. Finally, the Marxist Political Economy studies the facts from a relatively recent category, financialization, where the accumulation model is based on finance. The financial sector extends to almost all sectors of economic life and this overwhelming penetration has contributed to the fragility of the system. The objective is to make a reading of the different approaches, together, to better understand the phenomenon under study.

Keywords: Great Recession; financial system; financial crisis; regulation; financialization. JEL Classification:E24, E44, F34, F36, B50.

\section{Introducción}

A inicios de 2008, cuando en Estados Unidos (EU) ya se apreciaban los efectos de la recesión que finalizaría con una tasa cero de crecimiento, el entonces secretario de Hacienda y Crédito Público en México, Agustín Carstens, dio una declaración muy peculiar acerca de la hecatombe económica por venir: "México tendrá un catarrito por la crisis en EU". Sin saberlo, las palabras del doctor en Economía por la Universidad de Chicago expresan claramente la situación imperante en la ciencia económica: nuestra capacidad de entendimiento es limitada. Los economistas contamos con muchos instrumentos para el análisis de la realidad: modelos, teorías, enfoques, econometría, matemáticas, etc. No obstante ello, no es raro emitir apreciaciones subjetivas por completo de los fenómenos estu- 
diados y, en particular, de las crisis. Inclusive, las consecuencias sociales de una crisis escasamente son objeto de estudio riguroso.

Los hechos demostraron al gobierno mexicano su visión equivocada pues el Producto Interno Bruto, la medida convencional de riqueza de un país, registró en el año 2009 una caída de 6.7\%y significó la pérdida de empleos, el aumento de la pobreza, así como el aumento de la inseguridad y de la descomposición social.

En EU, epicentro de la crisis, se deterioró el concepto del estilo de vida americano (Rapoport y Brenta, 2010), es decir, muchas familias quedaron en la ruina y perdieron sus bienes. Desde su idiosincrasia, para muchos estadounidenses en situación crítica se concebía mejor la muerte que vivir sin posesiones materiales.

¿Qué fue lo que sucedió en 2008-2009? Primero, la academia ha catalogado el hecho como la Gran Recesión porque es la mayor caída que ha experimentado el capitalismo ${ }^{3}$, incluso mayor que la crisis de 1929 que se estudia como un evento muy lejano en los libros de historia económica. Y aun, desde una perspectiva teórica, Guillén (2015), Corsi (2010), Márquez (2010) y Foster y Magdoff (2010) conciben que el cisma fue resultado de factores estructurales de largo plazo que se reflejaron en la disminución secular de las tasas de crecimiento económico, el aumento de la fragilidad e inestabilidad financiera. De esta forma, la crisis de 2008-2009 no se ha resuelto y continúan sintiéndose sus efectos.

En segundo lugar, el título del texto incluye dos fechas ya que en 2008 es cuando explota la crisis en EU y en 2009 se extiende a la mayor parte del mundo. Algunos autores mencionan que aún estamos en recuperación y recobrar las condiciones imperantes previas demorará hasta 10 años. Incluso puede ser un lapso mayor en función de las acciones que se apliquen en las distintas economías implicadas.

En tercer lugar, la Gran Recesión es un suceso que inicia en el sector financiero por la falta de regulación y se extiende a lo largo de la economía de las empresas y los hogares (economía real). En específico,

\footnotetext{
3 Desde un punto de vista cuantitativo, para Turizo y Primo (2017) y De la Luz et al. (2015) el impacto inicial de la crisis de 2008 fue más intenso que el provocado por el de 1929; la tasa de desempleo en EU en 1929 fue de 3.1\% y en 2008 ascendió a $8.1 \%$ y el crecimiento del PIB, para el mismo país y para los mismos años, fue de $6.11 \%$ y $1.8 \%$.
} 
se otorgaron créditos hipotecarios a personas sin solvencia económica para pagar el préstamo, entonces los acreedores vendieron esos títulos de deuda a otros agentes financieros, como bancos de inversión. Nótese que esas deudas pasaron de personas expertas en el giro a agentes primerizos en el manejo de este tipo de instrumentos. El proceso continuó, los bancos de inversión también intercambiaron los títulos con otras instituciones, algunas de ellas no bancarias. Se consolidó una pirámide con cimientos muy endebles, incluso se llegó a falsear información, de tal forma que los ladrillos para la construcción resultaron piezas de barro. Las calificadoras asignaban puntajes altos a los títulos tóxicos; mintieron.

En suma, los títulos de deuda pasaron por muchas manos llegando al límite cuando el acreedor hipotecario se desconectó del deudor, esto continuó así hasta que los prestatarios cayeron en insolvencia. A inicios de 2007 estalla la burbuja hipotecaria, cae el mercado inmobiliario junto con el mercado financiero, se extiende a la economía y, en cuestión de meses, se esparce al resto del mundo. La Gran Recesión estaba presente: el 15 de septiembre de 2008 Lehman Brothers, el cuarto banco más grande de EU se hundía.

En plena contienda electoral, el gobierno de EU activó la intervención gubernamental para enfrentar la quiebra de las grandes empresas caracterizadas como "toobig to fall". El dinero fluyó a los bancos para su rescate en octubre de 2008 y en diciembre esas instituciones amparadas se aprobaron bonos navideños estratosféricos.

Surgen varias preguntas que aún son tema de discusión en la academia desde el inicio de la mayor crisis del capitalismo: ¿Por qué ocurrieron así los hechos? ¿Por qué el gobierno fue tan laxo y omiso? ¿Por qué los economistas no se pronunciaron enérgicamente para el cambio de agenda de investigación? Explicar satisfactoriamente los hechos sucedidos en 2008, es una deuda pendiente con la sociedad. Para realizar esa tarea, desde la ciencia económica se hacen diferentes interpretaciones teóricas que proporcionan explicaciones más o menos lógicas de lo acontecido y de las posibles consecuencias sociales. No obstante, cabe resaltar que las mismas no sólo no son coincidentes sino, aún más, son contradictorias. 
La idea aquí es hacer una lectura de conjunto de las interpretaciones más relevantes a fin de tener una mejor comprensión de los eventos sucedidos.

El texto está conformado por tres secciones, en la primera se plantea el contexto desde la mirada de largo plazo de la historia económica. En la segunda parte se analiza la posición de la teoría económica, convencional y heterodoxa. En la última sección se hace un acercamiento a la posición de la Economía Política Marxista. El documento finaliza con la exposición de algunas conclusiones.

\section{El contexto, el enfoque desde la historia económica}

El estudio del siglo XX, desde la perspectiva de los historiadores, se basa en tres eventos de magnitud considerable: la época de las catástrofes, la edad de oro y la época de la descomposición; en lo que Hobsbawm (1998) cataloga como un siglo corto.

En la primera etapa se ubican las dos guerras mundiales y el mundo capitalista presenció la crisis conocida como la Gran Depresión en 1929. Fue un periodo en donde apremió la falta de ideas prácticas para aplicar medidas eficientes de política económica y generar certidumbre, en todos los sentidos, a la sociedad. Entre los hechos que lo caracterizan se tienen: una esperanza de vida estancada, reducido acceso de la población joven a estudios de tiempo completo y no existían prestaciones sociales como el salario indirecto (seguro de desempleo, infraestructura escolar, fondo de vivienda, entre otros).

En la segunda etapa, la edad de oro, a la par de un desarrollo económico sin precedentes en el cual mejoraron sustancialmente las condiciones de vida en las diferentes economías que conformarían el mundo desarrollado, se observó un crecimiento sostenido de la población y el cambio estructural en su composición de rural a urbana. Procesos que, entre otros aspectos, posibilitaron el acceso masivo a bienes y servicios nuevos (automóvil, vivienda, enseres domésticos -refrigeradores, lavadoras, televisión, etc.-; educación superior, periodos vacacionales programados a playas, montañas e interurbanos).La conformación de una economía mixta encabezada por EU está en la base de este gran crecimiento, de acuerdo con el historiador Eric Hobsbawm (1998). 
En la última etapa, la descomposición, se agudizaron contradicciones dentro de las diferentes versiones del capitalismo; es decir, mientras que los países centrales o desarrollados mantenían el modelo en constante crecimiento, en los países tercermundistas o en vías de desarrollo se manifestaron problemas económicos (inflación, desempleo, distribución del ingreso, deuda ${ }^{4}$ y sociales -libertad de expresión, analfabetismo, rezago educativo, salud-. Además, el conflicto entre las dos potencias centrales, EU y la URSS, se agudizó en la segunda fase de la guerra fría; ocasionando en el campo socialista europeo caos político, posteriormente financiero y finalmente su disolución desembocando en lo que en ese momento se designó como tránsito al capitalismo salvaje, o también llamado desorganizado, donde se impulsó la desregulación total de la economía, las privatizaciones y los fundamentalismos de la libre acción de las leyes del mercado (Rojas, 2006).

Ambos fenómenos repercutieron negativamente en el modo de acumulación entrampando la expansión mundial de capital, lo cual llevó a la implementación de políticas de ajuste estructural que, entre otros efectos, pusieron a disposición del capital abundante fuerza de trabajo barata, recursos naturales y empresas rentables para la obtención de ganancias extraordinarias (Sierra, 2014; Márquez,2010; Fair, 2008).

En el marco de este este escenario disruptivo se impuso una forma de ver y entender el mundo: el neoliberalismo, cuya propuesta original puede rastrearse en Adam Smith ${ }^{5}$ y David Ricardo ${ }^{6}$, por mencionar a los autores más destacados de la economía clásica. El neoliberalismo es una teoría que propone promover el bienestar del ser humano por medio de

\footnotetext{
4 En forma lineal, Reinhart y Rogoff (2008) exponen que regularmente las crisis de deuda global se expanden desde los países centrales por medio de los precios de los productos básicos, los flujos de capital, las tasas de interés y los choques para la confianza de los inversores. Empero, no cabe aquí una interpretación inversa, por lo cual la crisis de 1995 en México -el llamado efecto tequila- y las subsiguientes en el sudeste asiático, Rusia y Latinoamérica no podrían explicarse.

5 "En la medida en que todo individuo procura en lo posible invertir su capital en la actividad nacional y orientar esa actividad para que su producción alcance el máximo valor, todo individuo necesariamente trabaja para hacer que el ingreso anual de la sociedad sea el máximo posible. Es verdad que por regla general él ni intenta promover el interés general ni sabeen qué medida lo está promoviendo.Al preferir dedicarse a la actividad nacional más que a la extranjera él sólo persigue su propia seguridad; y al orientar esa actividad de manera de producir un valor máximo él busca sólo su propio beneficio, pero en este caso como en otros una mano invisible lo conduce a promover un objetivo que no entraba en sus propósitos.....(Smith, 1996,p. 554).

6 "En un sistema de intercambio perfectamente libre, cada país dedicará lógicamente su capital y su trabajo a aquellas producciones que son las más beneficiosas para él. Pero este propósito de perseguir la ventaja individual está admirablemente unido a la conveniencia general del conjunto....(Ricardo, 2003,p. 115).
} 
la libertad, entendida tanto en las prácticas ${ }^{7}$ como en el pensamiento político-económico.

El neoliberalismo, en la práctica, tiene una característica principal: limitar la intervención económica del Estado porque su actuar condiciona la libertad individual (Vargas, 2007). Con lo cual el modelo de acumulación impuesto ha favorecido la distribución a través de los mecanismos de mercado. A perspectiva, esto ha significado, por lo menos en países en vías de desarrollo, que las clases superiores no tuvieran límites para aumentar su poder e incrementar su ingreso con consecuencias desastrosas para las clases populares (Duménil y Lévy, 2011); en otras palabras, un estancamiento del crecimiento económico y un aumento de la desigualdad.

Para Hobsbawm (2011) este periodo, aún en estudio, es una manifestación presente y futura de una tendencia de largo plazo y es poco probable que el capitalismo encuentre mecanismos para configurar un camino alternativo favorable para la humanidad en su conjunto. En esta óptica, la salida de la crisis de 2008-2009 muestra que el rumbo político, social y financiero de los países no diferirá gran cosa en sus líneas generales: fortalecimiento/concentración de la riqueza en el mundo desarrollado (Piketty, 2014) y agudización de la desigualdad en los países tercermundistas o en vías de desarrollo. En el mismo sentido la Organización para la Cooperación y el Desarrollo Económico reconoce que la movilidad social se ha estancado en las últimas tres décadas, es decir, los ricos y pobres son más propensos a permanecer en esa condición (OCDE, 2018).

\section{Perspectiva de la teoría económica}

\subsection{La economía convencional}

Dentro de la teoría económica ortodoxa hablar de crisis no es un tema atractivo (Gutiérrez, 2017), muchos economistas que marcan agenda de investigación enfatizaban que el tema predominante en el nuevo

\footnotetext{
7 "El papel del Estado es crear y preservar el marco institucional apropiado para el desarrollo de éstas prácticas. Por ejemplo, tiene que garantizar la calidad y la integridad del dinero. Igualmente, debe disponer las funciones y estructuras militares, defensivas, policiales y legales que son necesarias para asegurar los derechos de propiedad privada y garantizar, en caso necesario mediante el uso de la fuerza, el correcto funcionamiento de los mercados. Por otro lado, en aquellas áreas en las que no existe mercado (como la tierra, el agua, la educación, la atención sanitaria, la seguridad social o la contaminación medioambiental), éste debe ser creado cuando sea necesario mediante la acción estatal...”. (Harvey, 2007,p. 6-7).
} 
milenio debía ser el crecimiento; por lo que el análisis del ciclo económico resultaba irrelevante (Rosende, 2009).

De hecho, antes de La Gran Recesión la mayoría de los economistas no habían percibido la existencia de fisuras en el paradigma convencional (Castañeda, 2010), siendo la más importante de ellas la concepción de equilibrio de mercado. Para Boyer (2010) una de las debilidades de este enfoque teórico es la centralidad del concepto de equilibrio, el cual se basa en un tiempo cinemático, es decir, se estudia el movimiento del equilibrio sin considerar las causas que lo originan.

Por su parte, Stiglitz $(2009,2010)$ explica las causas del evento calificándolas decomplejas y simples a la vez. Los determinantes son simples al entender cómo la operación de los instrumentos financieros generaron una burbuja inmobiliaria. El fenómeno también es complejo desde la óptica de la teoría económica pues la crisis vino a mostrar la falta de operatividad de muchos supuestos como expectativas racionales, equilibrio, mercados eficientes, etc.

Para algunos economistas ortodoxos, luego de percibir las repercusiones sociales (Katkov, 2012; Verick y Islam, 2010) así como la caída del consumo, inversión privada y flujos de crédito de esta reciente crisis, la conclusión es clara: el laissez faire no era una opción de política económica(Blinder y Zandi, 2010). Sin embargo, para otros autores fue la falta de libertad lo que ocasionó la hecatombe económica: la regulación existía y existe pero no sólo no ha evitado la crisis sino que ha contribuido a generarla y agravarla (Sala-I-Martín, 2008). Su respuesta es fomentar los mecanismos de mercado.

En tanto Krugman (2009), un economista disidente del esquema ortodoxo, reconoce que fue acertado el uso del viejo estímulo keynesiano para reactivar el crédito. Matizando el comentario, la política del gobierno de EU se ha catalogado como keynesiana. Sin embargo, el rescate bancario es sumamente anti keynesiano (Marshall, 2011) pues los recursos no se destinaron a proyectos productivos, antes bien al pago de bonos de productividad de navidad y fin de año.

En el análisis de las crisis de 1929 y 2008 se acepta que el sistema financiero fue el factor clave de las mismas dado el mal diseño y fragilidad 
del sistema bancario, puesto que ahí no solo se originó, sino también se prolongó e intensificó la crisis financiera (Eigner y Umlauft, 2015). Ahora la discusión gira en torno a cómo plantear una reforma para superarlas debilidades que provocaron la última crisis (Krugman, 2009) y crear políticas redistributivas para evitar que el ciclo afecte severamente la demanda efectiva en el componente consumo(Stockhammer, 2012; Vilella, 2018). Pues una característica clave de los autores neokeynesianos y postkeynesianos es la importancia de la demanda para definir las políticas a corto y largo plazo (Lavoie, 2015).

\subsection{Postkeynesianos}

La teoría postkeynesiana asume un enfoque crítico a los posicionamientos de la teoría convencional. Por ejemplo, Lavoie (2018) comenta que la teoría ortodoxa se expía a si misma dado que para ésta el mercado no tiene nada que ver con la crisis financiera de 2008. Esto es así porque existe la creencia de que el modelo macroeconómico ad hocno consideró el papel del sistema financiero (Ireland 2010), por lo cual la solución reside en incorporar las fricciones del sistema bancario a los modelos de equilibrio general.

En esta coyuntura política y económica, la insatisfacción actual con la economía convencional sin duda proporciona una oportunidad para los postkeynesianos pues aporta elementos para plantear una reforma a la teoría y política económicas (Whalen, 2012); pero dejando atrás la reacción ante la postura convencional a fin de lograr un acercamiento y diálogo constructivo entre los dos enfoques teóricos (Keen, 2013). En palabras de Seidman (2012), los economistas deben lanzar una segunda revolución keynesiana, ahora con el calificativo de postkeynesiana.

Así, los postkeynesianos apoyan la regulación de los mercados financieros, pero ¿cómo debe efectuarse esa reforma? En lo particular, la respuesta es clara, Arestis (2013)explica que se deben eliminar los subsidios para las instituciones financieras; las empresas deben implementar sus propios mecanismos para generar riqueza y no buscar externalidades que proporcionen ganancias exógenas; otra medida es impulsar la demanda de forma sostenida en un contexto de maximización del bienestar social (Arestis, 2012).En ese sentido, es insuficiente la investigación que enfatiza el análisis sobre los efectos dinámicos de la crisis en la demanda (Ball, 
2014), es decir, determinar si los efectos de histéresis son reversibles y/o permanentes. La histéresis es un concepto relativamente nuevo en la agenda de investigación de los postkeynesianos, por ello no existe una definición única. En lo general, el concepto se refiere a las afectaciones en las variables provocadas por los cambios dentro de un sistema, sin que el restablecimiento de las condiciones normales implique el regresa al estado original. Los efectos de la histéresis se estudian, principalmente, en los mercados de trabajo y cambiario. Por ejemplo, en el mercado laboral, los trabajadores pierden sus empleos por los ajustes realizados por las empresas en las crisis económicas y estos desempleados ya no pueden colocarse en sus antiguas posiciones; pierden habilidades laborales que no recuperan.

En lo general, la discusión se centra en identificar a los agentes que detonaron la Gran Recesión. Dos interpretaciones son las más socorridas: en la estándar los villanos son los banqueros y en la interpretación monetaria la culpa se atribuye a los reguladores (Congdon, 2017). Es un asunto que sigue esculpiéndose en la academia. La primera interpretación se enfoca en una explicación netamente financiera por medio de la hipótesis de inestabilidad financiera de Hyman Minsky (economistas de la teoría del dinero moderno o MMT por sus siglas en ingles cuyo principal exponente es Randall Wray). La segunda proposición proviene de los keynesianos estructurales, su supuesto principal se basa en el estancamiento de los salarios y el aumento de la desigualdad en los ingresos como detonantes de la crisis financiera, es decir, las causas de la crisis son reales (Palley, 2019).

Lo cierto es que se deben tomar medidas estructurales (Cowling et al., 2011; Boyer, 2009)y los gobiernos deberán ser más cuidadosos de la reacción que desencadena la aplicación de los instrumentos de política económica, ya que aun cuando se haya aprendido de los eventos del pasado, la Gran Depresión y la Gran Recesión no son eventos iguales (Lavoie y Seccareccia, 2016; Faverzani et al., 2017). También es necesario controlar al sistema bancario por medio de una regulación más estricta y en constante actualización, pues el sistema financiero es por naturaleza inestable(Sawyer, 2011) dada la innovación permanente en la búsqueda de mecanismos para la optimización de la tasa de ganancia. 


\section{La posición de la Economía Política Marxista}

En la interpretación de la crisis por parte de los marxistas no hay una versión homogénea, pudiéndose identificar dos orientaciones. En la primera exégesis se hace referencia a las ideas de Karl Marx en El Capital, en donde el aumento de la productividad, junto con las innovaciones tecnológicas, ahorrará trabajo y reducirá la tasa promedio de ganancia (Smith, 2010), fenómeno caracterizado como ley de la tendencia decreciente de la tasa de ganancia que afecta a todo el sistema capitalista.

Dicha ley es central en la teoría de las crisis pues ellas son resultado de una contradicción entre la producción física y la de valor en el funcionamiento mismo del capitalismo; es decir, a medida que la productividad física aumenta, el valor individual de las mercancías disminuye (Kliman, 2015), en consecuencia, la disminución de la participación del capital variable y el aumento del capital constante en el proceso de producción redunda en una caída de la tasa de ganancia.

Para Desai y Freeman (2011), la productividad aumenta de tal forma que contrarresta la ley de la competencia y la acumulación por lo que la caída de la ganancia sólo se produce en periodos cortos. Además, esta visión no se encuentra articulada con el desarrollo actual del sistema financiero dado que en el análisis hecho por Marx, las finanzas no ocupaban un lugar preponderante en la dinámica de la ganancia.

La segunda posición propone el análisis con base en las categorías de capital ficticio y financiarización. El capital ficticio es un concepto sui géneris dentro de la teoría económica que se presenta como la síntesis de las formas autonomizadas funcionales del capital que devenga interés (Alcalá, 2015); en tanto la financiarización se reconoce en la figura de capital especulativo (Lechuga y Bustamante, 2008).Para Cédric Durand (2017), el capital ficticio se caracteriza por la acumulación y comercio de acciones, derivados y otros instrumentos financieros. De esta forma el capital ficticio se desarrolla en términos aparentemente autónomos que facilitan el surgimiento de burbujas especulativas y productos derivados sofisticados (Taques et al., 2017). Por lo tanto, el sistema bancario es el epicentro de la crisis (Ivanova, 2013). 
El término financiarización tiene muchas acepciones y su análisis se ha robustecido en la época contemporánea (Vega, 2017; Rodrigues y Palludeto, 2018; Vega et al., 2019). Así, Ramos (2017) proporciona una interesante categorización de los enfoques de estudio: la relación cambiante entre las finanzas y otros sectores económicos, los cambios dentro del sistema financiero y la creciente magnitud de las finanzas a nivel internacional. La literatura marxista se centra en la tercera categoría.

En ese sentido, para este enfoque teórico la financiarización se relaciona con la caracterización del modelo de acumulación a partir de las finanzas (Mateo, 2015), es decir, el giro de la producción de mercancías al uso cada vez más fuerte de los canales financieros por parte de las empresas (Raghavendra, 2018).

A su vez, desde el enfoque de la financiarización existen dos orientaciones que se contraponen. Para Arturo Guillén (2015) el fenómeno de la financiarización no es nuevo ya que el capitalismo ha experimentado varios eventos de la misma naturaleza con características particulares, desde la implantación del imperialismo moderno pasando por la caída de la regulación del capital monopolista hasta la crisis de 2008-2009. En este periodo, la financiarización es un mecanismo activo-pasivo que emplean las potencias económicas para encontrar salidas de las grandes crisis. Por ejemplo, la financiarización toma un rol pasivo cuando las condiciones de producción son estables, pero cuando se interrumpe la acumulación como en las décadas de los 20’s y 70`s, el capital se traslada a las finanzas para valorizarse y la financiarización tiene un rol protagónico. Por lo tanto, la crisis actual es resultado de la falta de regulación y también de los procesos de transición hegemónica.

En la misma tónica, Héctor Guillén (2009a) propone que las finanzas siempre han estado presentes por medio de las innovaciones, lo cual suscita el entusiasmo para invertir más recursos y la finanza, como la llama este autor, ha contribuido a la fragilidad del sistema (Guillén, 2009b). Actualmente, la finanza se coloca en una posición superior a la esfera productiva y asegura una rentabilidad financiera estricta, consagrada por retribuciones generosas a los accionistas y ganancias importantes en la bolsa (Guillén, 2015). 
Una segunda mirada de la financiarización la provee Lapavitsas (2016), quien realiza una revisión de la literatura marxista sobre el concepto y propone su análisis con base en El capital financiero de Rudolf Hilferding y El imperialismo de Vladimir Lenin. El autor encuentra que las finanzas siempre han tenido una actuación fundamental en el desarrollo del capitalismo. Empero el rasgo característico de la década de los 70`s es la expansión de las finanzas por medio de empresas no financieras, bancos y hogares en la búsqueda de mayores ganancias, de ganancias exógenas. Así como la creación de plusvalía era el rasgo definitorio del capitalismo de libre concurrencia, la financiarización es el rostro de un trastorno financiero sistémico que ha arrojado luz sobre la trayectoria del desarrollo social y económico del capitalismo contemporáneo.

Independientemente de la concepción, lo importante es entender la categoría financiarización como hilo conductor para explicar la crisis de 2008-2009. Dymski (2011) reconoce cuatro raíces estructurales que la detonaron: el contexto macroeconómico de Estados Unidos con alta nivel de liquidez, la transformación de las estrategias bancarias para la generación de utilidades, un escenario de inclusión financiera y otorgamiento de préstamos a los hogares y el prolongado descenso de los salarios de la clase trabajadora estadounidense.

Esta última causa es la base de la explicación de Lapavitsas (2009), asumiendo que las finanzas se encuentran tan relacionadas con la vida cotidiana que los bancos han tomado los ingresos de los trabajadores como fuente de ganancias, de tal forma que los salarios han entrado al proceso de financiarización o expropiación financiera (Lapavitsas, 2011).

Los salarios se habían rezagado con bastante antelación, por lo cual no constituían un adecuado fundamento para la generación de ganancias para los bancos, así que las instituciones generaron innovaciones financieras no vinculadas con la solvencia real de los depositantes (ahorradores) y/o los demandantes de crédito. La creciente precariedad en los ingresos empujaba a contraer mayores deudas para mantener los niveles de consumo y el punto de quiebre de este mecanismo acaeció cuando los sujetos de crédito ya no tuvieron recursos para solventar sus deudas, particularmente hipotecarias. El estallido de la acumulación de deuda resultaba insostenible e inminente y al poco tiempo se reflejaron sus efectos en toda la economía (Sawyer, 2018). 


\section{Reflexiones Finales}

En la época dorada del capitalismo (1945-1970) el sistema mostró que tenía mecanismos por los cuales las economías desarrolladas podían crecer sin presiones inflacionarias con una equilibrada distribución del ingreso. No obstante, contradicciones del modelo benefactor o desarrollista confluyeron para el cambio de paradigma, desembocando en el neoliberalismo.

La nueva estrategia de crecimiento tiene muchas características interesantes para el análisis, de las más importantes resalta la restricción de la intervención del Estado en la vida pública de las sociedades, basada en la libertad de mercado.

La crisis de 2008, para algunos autores, abría la posibilidad de un cambio de agenda de política económica en los principales países. Empero las acciones de los principales agentes muestra que no fue, ni será así; al menos hasta el largo plazo.

En el presente trabajo no se ha buscado ofrecer una cronología de los eventos de la gran crisis, sino identificar las visiones teóricas del evento. La revisión inicia con la postura de la teoría económica, convencional y heterodoxa, hasta el examen de los planteamientos marxistas.

Así, para la ortodoxia pura, la crisis fue resultado del exceso de regulación, por lo que su recomendación es fomentar la libertad de mercado pues dentro de la lógica neoclásica las crisis son fenómenos exógenos. Sin embargo, esta visión es parcial al seguir apoyándose en el fundamentalismo del mercado como el mecanismo ad hoc para la toma de decisiones económicas; es claro que de ser cierta esta idea no habría estallado la crisis pues mayor desregulación financiera no había operado desde los años dorados del capitalismo. Los modelos ortodoxos carecen de operatividad, siendo ellos precisamente la base para construir los modelos de política económica, siendo abstracciones sin vinculación con la realidad.

Los enfoques heterodoxos -postkeynesianos- se han caracterizado por la falta de un programa homogéneo, lo único que los une es la critica a la teoría convencional; recalcando que, en la medida en que los instrumentos empíricos no contribuyen a la comprensión de la realidad, menos de la crisis, quedan enclaustrados en este razonamiento. El caso más rele- 
vante es la supuesta "robustez" del enfoque econométrico en la predictibilidad del comportamiento de las variables económicas, dado que no fue una herramienta eficaz para prever, ni luego para explicar, las causas de fondo de la Gran Recesión. Y si bien es cierto que el enfoque postkeynesiano reclama, con cierta justicia, la regulación de los mercados financieros, se limita a este aspecto y omite que las crisis del sistema capitalista son de carácter endógeno.

Finalmente, en la Economía Política Marxista hay un cierto aereamiento teórico al proponer una nueva categoría de análisis, financiarización, entendida como un desbordamiento de las finanzas en el proceso de acumulación. Proceso en el cual el sector financiero sustenta sus ganancias en la apropiación de valor sin un respaldo sólido y real en la creación del mismo.

Las observaciones de la Economía Política Marxista son consistentes pues proponen un estudio pormenorizado inclusivo de los detonantes reales y financieros de la crisis, analiza sus posibles repercusiones y, en ese sentido, supera las propuestas anteriores.

Lo aquí presentado no pretende ser una versión homogénea de las propuestas teóricas referidas; más bien la lectura que se debe hacer es la de partes independientes que pudieran complementarse para explicar $L a$ Gran Recesión, fenómeno aún en estudio.

\section{Referencias Bibliográficas}

Alcalá, Leinad (2015). La lógica interna de valorización del capital. Un análisis crítico de la categoría capital ficticio. Revista Cuadernos Economía, Vol. 1, No. 1, p. 37-50.

Arestis, Philip (2012). Fiscal policy: a strong macroeconomic role. Review of Keynesian Economics, Inaugural Issue, p. 93-108.

Arestis, Philip (2013). Economic theory and policy: a coherent post-Keynesian approach. European Journal of Economics and Economic Policies: Intervention, Vol. 10 No. 2, p. 243-255.

Ball, Laurence (2014). Long-term damage from the Great Recession in OECD countries. European Journal of Economics and Economic Policies: Intervention, Vol. 11, No. 2, p. 149-160. 
Blinder, Alan y Mark Zandi (2010). How the Great Recession Was Brought to an End.

Boyer, Marcel (2009). La crise économique et ses conséquences sur l'emploi. Montreal: Institut économique de Montréal. 29p.

Boyer, Robert (2010). La crisis actual a la luz de los grandes autores de la Economía Política. Revista Economía: Teoría y Práctica, Vol. NE, No. 33, p. 11-58.

Castañeda, Gonzalo (2010). Crisis económicas y cambios de paradigma. RevistaEstudiosEconómicos, Vol. 25, No. 2, p. 425-441.

Congdon, Tim (2017). What were the causes of the Great Recession? The mainstream approach vs. the monetary interpretation. En Money in the Great Recession. Did a Crash in Money Growth Cause the Global Slump? Buckingham Studies in Money, Banking and Central Banking.ReinoUnido: Edward Elgar Pub. 288p.

Corsi, Francisco. (2010). La crisis estructural del capitalismo y sus repercusiones. Íconos, Revista de Ciencias Sociales, Vol. NE, No. 36, p. 29-39.

Cowling, Keithet al. (2011). Global imbalances and modern capitalism: a structural approach to understanding the present economic crisis. Journal of Post Keynesian Economics, Vol. 33, No. 4, p. 575-596.

De la Luz, Gloriaet al. (2015). La crisis financiera internacional de 2008 y algunos de sus efectos económicos sobre México. Revista de Contaduría y Administración, Vol. 60, No. 2, p. 128-146.

Desai, Radhika y Alan Freeman (2011). Value and Crisis Theory in the 'Great Recession'. Munich Personal RePEc Archive, Documento de trabajo No. 48645.

Duménil, Gérard y Dominique Lévy (2011). A crise do neoliberalismo na história do capitalismo. 2008-2011, os doisprimeirosatos.

Durand, Cédric (2017). Fictitious capital. Howfinanceisapprpriatingourfuture. Nueva York: Verso Books. 176p.

Dymski, Gary (2011). La exclusión racial y la economía política. En Morera, Carlos y Costas Lapavitsas (Coordinadores). La crisis de la financiarización. México: UNAM, Instituto de Investigaciones Económicas, Consejo Latinoamericano de Ciencias Sociales. 400p.

Eigner, Peter y Thomas Umlauft (2015). The Great Depression(s)? Of 1929-1933 and 2007-2009. Parallels, differences and policy lessons. Hungarian Academy of Science MTA-ELTE Crisis History, Documento de trabajo No. 2.

Fair, Hernán. (2008). El sistema global neoliberal”. Revista Latinoamericana Polis, Vol. NE, No. 21, p. 1-30. 
Faverzani, Henriqueet al. (2017). A criseeconômica mundial de 2008 e o reflexo sobre as instituiçõesbancárias brasileiras. Estudo\& Debate, Vol. 24, No. 1, p. 26-41.

Foster, John y Fred Magdoff (2010). The Great Financial Crisis-Three Years On. Monthly Review, Vol. 62, No. 5, p. 52-55.

Guillén, Arturo(2015). La crisis global en su laberinto. México: Consejo Editorial de la División de Ciencias Sociales y Humanidades, UAM Iztapalapa. 285p.

Guillén, Héctor(2009a). Kindleberger y Galbraith: el eterno retorno de las crisis financieras". Revista Mundo siglo XXI, Vol. NE, No. 18, p. 15-25.

Guillén, Héctor (2009b). La crisis financiera de los préstamos subprime. Revista de Comercio Exterior, Vol. 59, No. 12, p. 947-969.

Guillén, Héctor(2015). Consideraciones en torno a la crisis contemporánea. Revista Economía UNAM, Vol. 12, No. 34, p. 31-52.

Gutiérrez, Arturo (2017). Las crisis económicas. Un acercamiento teórico. Revista Economía y Desarrollo, Vol. 158, No. 1, p. 6-16.

Harvey, David (2005). Breve historia del neoliberalismo. Madrid: Ediciones Akal. 252p.

Hobsbawm, Eric (1998). Historia del siglo XX. Buenos Aires: Editorial Crítica. $610 \mathrm{p}$.

Hobsbawm, Eric (2011). How to change the world? Reflections on Marx and Marxism. Baskerville: Yale University Press. 470p.

Ireland, Peter (2010). A New Keynesian Perspective on the Great Recession. National Bureau of Economic Research, Documento de trabajo No. 16420

Ivanova, Maria (2013). Marx, Minsky, and the Great Recession. Review of Radical Political Economics, Vol. 45, No. 1, p. 59-75.

Katkov, Alexander (2012). Great Recession of 2008-2009: Causes and Consequences. Journal of Applied Business and Economics, Vol. 13, No. 3, p. 107-122.

Keen, Steve (2013). Predicting the 'Global Financial Crisis': Post-Keynesian Macroeconomics. Economic Record, Vol. 89, No. 285, p. 228-254.

Kliman, Andrew (2015). The Great Recession and Marx's crisis theory". American Journal of Economics and Sociology, Vol. 74, No. 2, p. 236-277.

Krugman, Paul (2009). Return of depres on Economics y crisis of 2008. Nueva York: W.W. Norton. 191p.

Lapavitsas, Costas (2009), Financialisedcapitalism: crisis and financialexpropriation. HistoricalMaterialism, Vol. NE, No. 17, p. 114-148. 
Lapavitsas, Costas (2011). Theorizing financialization. Work, employment and society, Vol. 25, No. 4, p. 611-626.

Lapavitsas, Costas (2016). Beneficios sin producción. Cómo nos explotan las finanzas. Madrid: Editorial Traficante de Sueños. 453p.

Lavoie, Marc (2015). What post-Keynesian economics has brought to an understanding of the Global Financial Crisis.

Lavoie, Marc (2018). Rethinking macroeconomic theory before the next crisis. Review of Keynesian Economics, Vol. 6, No. 1, p. 1-21.

Lavoie, Marc y Mario Seccareccia (2016). Understanding the Great Recession: Some Fundamental Keynesian and Post-Keynesian Insights, with an Analysis of Possible Mechanisms to Achieve a Sustained Recovery. Institutefor New EconomicThinking, Documento de trabajo No. 37.

Lechuga, Jesús y Jorge Bustamante (2008). Estado y bancos en la creación monetaria. El valor del dinero electrónico. Revista Trayectorias, Vol. 10, No. 27, p. 35-46.

Márquez, Humberto (2010). Crisis del sistema capitalista mundial: paradojas y respuestas. Polis, Revista de la Universidad Bolivariana, Vol. 9, No. 27, p. 435461.

Márquez, Humberto (2010). La gran crisis del capitalismo neoliberal. Revista Andamios, Vol. 7, No. 13, p. 57-84.

Marshall, Wesley (2011). La Reserva Federal frente a la crisis y sus efectos sobre el sistema financiero global. Revista Ola Financiera, Vol. 4, No. 9, p. 58-89.

Mateo, Juan (2015). La financiarización como teoría de la crisis en perspectiva histórica. Revista Cuadernos de Economía, Vol. 34, No. 64, p. 23-44.

Organización para la Cooperación y el Desarrollo Económico -OCDE (2018). A Broken Social Elevator? How to Promote Social Mobility. Overview and main findings.Paris. 61p.

Palley, Thomas (2019). What's wrong with modern money theory (MMT): A critical primer". ForumforMacroeconomics and MacroeconomicPolicies, Documento de trabajo No. 44.

Piketty, Thomas (2014). El capital en el siglo XXI. Buenos Aires: Fondo de Cultura Económica. 679p.

Raghavendra, Srinivas (2018). The principle of effective demand under modern finance: an exploration in the traditions of Kalecki and Keynes. Panoeconomicus, Vol. 65, No. 3, p. 267-287. 
Ramos, Raquel (2017). Financialization at the international level: evidence from emerging market economies. Economia e Sociedade, Vol. 26, No. especial, p. 959-990.

Rapoport, Mario y Noemí Brenta (2010). La crisis económica mundial: ¿el desenlace de 40 años de inestabilidad?. Revista Problemas del Desarrollo, Vol. 163, No. 41, p. 7-30.

Reinhart, Carmen y Kenneth Rogoff (2008). This time is different: a panoramic view of eight centuries of financial crises. National Bureau of Economic Research, Documento de trabajo No. 13882.

Ricardo, David (2003). Principios de Economía Política y Tributación. Madrid: Ediciones Pirámide. 356p.

Rodrigues, André y Alex Palludeto (2018). Themacroeconomics of financialization: a bibliometricsurvey.

Rojas, Jorge (2006). La sociedad neoliberal. Revista Sociedad Hoy, Vol. NE, No. 10, p. $41-72$.

Rosende, Francisco (2009). La macroeconomía y la crisis: ¿La crisis de la macroeconomía?. Revista Cuadernos de Economía, Vol. 46, No. 134, p. 161-182.

Sala-I-Martín, Xavier (2008). Crisis Financiera (3): Neo-Intervencionismo.

Sawyer, Malcolm (2011). 'It' keeps almost happening: post-keynesian perspectives on the financial crisis and the Great Recession. History of Economic Ideas, Vol. 19, No. 2, p. 149-164.

Sawyer, Malcolm (2018). Financialisation, financial crisis and inequality.

Seidman, Laurence (2012). Keynesian stimulus versus classical austerity. Review of KeynesianEconomics, Vol. NE, No. inaugural, p. 77-92.

Sierra, Yoandris (2014). Análisis del cambio del modelo global de acumulación capitalista sucedido en la década de los años setenta del siglo XX. Elementos a observar ante un posible cambio. Revista Economía y Desarrollo, Vol. 152, No. 2, p. 6-19.

Smith, Adam (1996). Adam Smith: La riqueza de las naciones (Libros I-II-III y selección de los libros IV y V).Madrid: Alianza Editorial. 818p.

Smith, Murray (2010). Global capitalism in crisis Karl Marx and the decay of the profit system. Halifax and Winnipeg: Fernwood Publishing. 192p.

Stiglitz, Joseph (2009). Interpreting the causes of the Great Recession of 2008. 
Stiglitz, Joseph (2010). Lessons from the Global Financial Crisis of 2008. Seoul Journal of Economics, Vol. 23, No. 3, p. 321-339.

Stockhammer, Engelbert (2012). Financialization, income distribution and the crisis. Revista InvestigaciónEconómica, Vol. 71, No. 279, p. 39-70.

Taques, Fernando et al. (2017). The 2007-08 Financial Crisis from a Marxist View. Modern Economy, Vol. NE, No. 8, p. 1069-1081.

Turizo, Héctor y Wilfrido Primo (2017). Análisis comparativo de las crisis financieras internacionales 1929 y 2008: una visión empresarial. Management Review, Vol. 2, No. 1, p. 1-19.

Vargas, José (2007). Liberalismo, Neoliberalismo, Postneoliberalismo. Revista del Magíster en Análisis Sistémico Aplicado a la Sociedad, Vol. NE, No. 17, p. 66-89.

Vega, Faustino (2017). Financiarización: un estudio desde la función consumo para México en el periodo 2000-2015. Revista Tiempo Económico, Vol. 12, No. 36, p. 59-75.

Vega, Faustino et al. (2019). Financiarización: la experiencia mexicana en el periodo 1993-2016. Revista Análisis Económico, Vol. 34, No. 85, p. 33-52.

Verick, Sher e Iyanatul. Islam (2010). The Great Recession of 2008-2009: Causes, Consequences and Policy Responses. The Institute for the Study of Labor (IZA), Documento de trabajo No. 4934

Vilella, Caio (2018). A Grande Recessão e a EscolaPós-Keynesiana: Umacomparação entre as vertentesminskyana e kaleckiana.

Whalen, Charles (2012). Post-Keynesian Institutionalism after the Great Recession. Levy Economics Institite of BardCollege, Documento de trabajo No. 724. 